\title{
Empadre de vicuñas en el cerco permanente del Centro de Investigación, Producción y Transferencia Tecnológica, CIPTT Tullpacancha - Universidad Alas Peruanas, Huancavelica - Perú
}

\author{
Breeding of vicunas in the permanent fence of the Center of Research, Production and \\ Technological Transfer in Tullpacancha - Alas Peruanas University in Huancavelica - Peru
}

\author{
Marcos Zúñiga ${ }^{1}$, Norma Bujaico ${ }^{2}$
}

http://dx.doi.org/10.21503/CienciayDesarrollo.2013.v16i2.02

\section{RESUMEN}

El propósito del presente estudio fue determinar los tiempos y las fases del empadre de las vicuñas del cerco permanente del CIPTT - TULLPACANCHA en los meses de marzo - abril del 2012 donde la población total es de 1700 vicuñas y la muestra de estudio fue de 182 ejemplares que habitan dentro del sector Pampa Corral. La muestra de la población estuvo dividido en 46 grupos familiares. Después de observar durante 36 días consecutivos en horas del día y de la noche se pudo determinar que existen dos fases bien definidas del empadre: El sometimiento y el coito propiamente dicho. Durante el día 12 vicuñas fueron sometidas en un tiempo promedio de 8.25 minutos y durante la noche 4 vicuñas con un tiempo promedio de 3.00 minutos. En cuanto al coito propiamente dicho en horas del día, se observó que el tiempo promedio utilizado para esta fase en 12 vicuñas, fue de 14.75 minutos y durante la noche para las 4 vicuñas fue de 28.50 minutos, dando como resultado para el empadre durante el día de 23.00 minutos y para la noche de 31.50 minutos en total.

Palabra clave: Empadre, sometimiento.

\section{ABSTRACT}

The purpose of this study was to determine the times and stages of the breeding season of the vicuñas of the permanent fence of the CIPTT - TULLPACANCHA in the months of March - April, 2012 where the total population is 1700 vicuñas and the study sample was 182 specimens inhabiting the Pampa Corral sector. The sample of the population was divided into 46 family groups. After observing for 36 consecutive days in day and night, it was determined that there are two well defined stages of breeding: submission and actual intercourse. On the 12th, vicunas were submitted in an average time of $8.25 \mathrm{mi}-$ nutes and during the night 4 vicuñas with an average time of 3.00 minutes. In relation to the intercourse strictly speaking in daytime, it was noted that the average time used for this stage in 12 vicuñas, was 14.75 minutes and overnight for 4 vicuñas was 28.50 minutes, resulting to the intercourse during the day of 23.00 minutes and to 31.50 minutes at night in total.

Keyword: Breeding, submission.

\footnotetext{
M.V. Investigador asesor CIPTT Tullpacancha.E-mail: anzuv20@hotmail.com, normisbm15@hotmail.com

Ingeniera zootecnista, Universidad Nacional de Huancavelica. E-mail: normisbm15@hotmail.com
} 


\section{INTRODUCCIÓN}

La vicuña (Vicugna vicugna) es un mamífero perteneciente a la familia camelidae cuya historia evolutiva empezó hace unos 40 millones de años en América del Norte. Es el más pequeño, grácil, escaso y fino ejemplar dentro de esta familia, la cual habita en la Cordillera de los Andes de Ecuador, Perú, Bolivia, Chile y Argentina entre los 3,800 y los 5,200 m.s.n.m. Esta especie se caracteriza fundamentalmente por ser silvestre ubicándose como un recurso altamente rentable.

Lo más relevante es la calidad de su fibra, ubicándola como una de las mejores, motivo por la cual su cotización en el mercado internacional es bastante alta, por lo tanto, urge la necesidad de buscar y asegurar la producción de la misma sin alterar las poblaciones y capacidad reproductiva de este camélido.

El objetivo de este estudio, fue determinar el comportamiento sexual de las vicuñas durante el empadre tanto en horas del día como en horas de la noche considerando sus dos fases definidas, el sometimiento y el coito propiamente dicho.

\section{MATERIALES Y MÉTODOS}

El presente estudio se realizó en el cerco permanente para vicuñas del Centro de Investigación Producción y Transferencia Tecnológica- Tullpacancha, ubicado en el distrito de Locroja provincia Churcampa Región Huancavelica - Perú, contando este con una población de 1700 ejemplares entre crías, hembras y machos.

Para la realización de este trabajo se observó una población de 182 vicuñas que habitan en el sector de Pampa Corral, la observación del comportamiento de los animales a investigar fue desde el 24 de Marzo hasta el 30 de Abril del 2012 coincidien- do con la época de lluvias y la misma época donde se lleva a cabo la parición.

Estas vicuñas están divididas en 46 grupos familiares, todas ubicadas dentro del cerco permanente.

El método utilizado fue la observación directa durante todas las horas del día y la noche donde se determinó el comportamiento del empadre de las vicuñas integrantes de los grupos familiares por espacio de 36 días.

De la población tomada como muestra, 12 vicuñas empadraron durante el día y 4 durante la noche donde pudimos observar las fases de sometimiento y copula.

\section{RESULTADOS Y DISCUSIÓN}

Durante el período estudiado se pudo determinar dos fases bien definidas.

La primera es la del sometimiento, fase en la cual el macho obliga a la hembra a tomar la posición de cúbito ventral.

Para las vicuñas que empadraron durante el día fue del tiempo más prolongado, esto obedece a que durante el día hay influencia de otras vicuñas entre crías y machos que interrumpen el sometimiento.

Durante la noche el empadre es mas corto, debido a la ausencia de luz natural y la quietud de los otros integrantes de los grupos familiares.

La segunda fase, es la cópula o coito propiamente dicho, el cual durante el día es más corto debido a la influencia de los integrantes de los grupos familiares, caso contrario que es durante la noche que es más prolongado debido a la quietud de la noche. 


\section{Tabla 1. Fases del empadre}

\begin{tabular}{|c|c|c|c|c|c|c|c|c|c|c|c|c|c|}
\hline FECHA & LUGAR & H.I. & H.T. & $\begin{array}{r}\mathrm{F} \\
\text { SOMET }\end{array}$ & SE & TTO & & & & FASE COPL & LA & & $\begin{array}{l}\text { TIEMPO } \\
\text { TOTAL }\end{array}$ \\
\hline $23 / 03$ & $\begin{array}{l}\text { Pampa } \\
\text { corral }\end{array}$ & 16.49 & 17.08 & 16.49 & - & 16.55 & $=$ & $0.06^{\prime}$ & 16.55 & 17.08 & $=$ & $0.14^{\prime}$ & 0.20' Diurno \\
\hline $24 / 03$ & $\begin{array}{l}\text { Pampa } \\
\text { corral }\end{array}$ & 08.54 & 09.13 & 08.54 & - & 07.30 & $=$ & $0.09^{\prime}$ & 09.03 & 09.13 & $=$ & $0.10^{\prime}$ & 0.19' Diurno \\
\hline $24 / 03$ & $\begin{array}{l}\text { Pampa } \\
\text { corral }\end{array}$ & 11.24 & 11.55 & 11.24 & - & 11.35 & $=$ & $0.11^{\prime}$ & 11.35 & 11.55 & $=$ & $0.20^{\prime}$ & 0.31' Diurno \\
\hline $25 / 03$ & $\begin{array}{l}\text { Pampa } \\
\text { corral }\end{array}$ & 09.17 & 09.44 & 09.17 & - & 09.21 & $=$ & $0.4^{\prime}$ & 09.21 & 09.44 & $=$ & $0.23^{\prime}$ & $0.27^{\prime}$ Diurno \\
\hline $25 / 03$ & $\begin{array}{l}\text { Pampa } \\
\text { corral }\end{array}$ & 11.01 & 11.28 & 11.01 & - & 11.20 & $=$ & $0.19^{\prime}$ & 11.20 & 11.28 & $=$ & $0.08^{\prime}$ & 0.27’ Diurno \\
\hline $28 / 03$ & $\begin{array}{l}\text { Pampa } \\
\text { corral }\end{array}$ & 15.33 & 15.41 & 15.33 & - & 15.37 & $=$ & $0.04^{\prime}$ & 15.37 & 15.41 & $=$ & $0.04^{\prime}$ & 0.08' Diurno \\
\hline $04 / 04$ & $\begin{array}{l}\text { Pampa } \\
\text { corral }\end{array}$ & 10.46 & 11.13 & 10.46 & - & 10.51 & $=$ & $0.05^{\prime}$ & 10.51 & 11.13 & $=$ & $0.22^{\prime}$ & $0.27^{\prime}$ Diurno \\
\hline $05 / 04$ & $\begin{array}{l}\text { Pampa } \\
\text { corral }\end{array}$ & 12.13 & 12.48 & 12.13 & - & 12.22 & $=$ & $0.09^{\prime}$ & 12.22 & 12.48 & $=$ & $0.26^{\prime}$ & $0.35^{\prime}$ Diurno \\
\hline $07 / 04$ & $\begin{array}{l}\text { Pampa } \\
\text { corral }\end{array}$ & 07.18 & 07.40 & 07.18 & - & 07.25 & $=$ & $0.07^{\prime}$ & 07.25 & 07.40 & $=$ & $0.15^{\prime}$ & 0.22' Diurno \\
\hline $10 / 04$ & $\begin{array}{l}\text { Pampa } \\
\text { corral }\end{array}$ & 09.11 & 09.31 & 09.11 & - & 09.18 & $=$ & $0.07^{\prime}$ & 09.18 & 09.31 & $=$ & $0.13^{\prime}$ & 0.20’ Diurno \\
\hline $10 / 04$ & $\begin{array}{l}\text { Pampa } \\
\text { corral }\end{array}$ & 13.52 & 14.10 & 13.52 & - & 14.01 & $=$ & $0.09^{\prime}$ & 14.01 & 14.10 & $=$ & $9^{\prime}$ & 0.18' Diurno \\
\hline $11 / 04$ & $\begin{array}{l}\text { Pampa } \\
\text { corral }\end{array}$ & 19.35 & 20.02 & 19.35 & - & 19.39 & $=$ & 0.04 & 19.39 & 20.02 & $=$ & $23^{\prime}$ & $0.27^{\prime}$ Nocturno \\
\hline $13 / 04$ & $\begin{array}{l}\text { Pampa } \\
\text { corral }\end{array}$ & 22.03 & 22.06 & 22.03 & - & 22.06 & $=$ & $0.03^{\prime}$ & 22.06 & 22.35 & $=$ & $29^{\prime}$ & 0.32 ' Nocturno \\
\hline $14 / 04$ & $\begin{array}{l}\text { Pampa } \\
\text { corral }\end{array}$ & 01.18 & 01.53 & 01.18 & - & 01.21 & $=$ & $0.03^{\prime}$ & 01.21 & 01.53 & $=$ & $32^{\prime}$ & 0.35' Nocturno \\
\hline $15 / 04$ & $\begin{array}{l}\text { Pampa } \\
\text { corral }\end{array}$ & 20.59 & 21.31 & 20.59 & - & 21.01 & $=$ & $0.02^{\prime}$ & 21.01 & 21.31 & $=$ & $30^{\prime}$ & 0.32' Nocturno \\
\hline $15 / 04$ & $\begin{array}{l}\text { Pampa } \\
\text { corral }\end{array}$ & 08.21 & 08.44 & 08.21 & - & 08.30 & $=$ & $0.09^{\prime}$ & 08.30 & 08.44 & $=$ & $0.14 ’$ & 0.23’ Diurno \\
\hline
\end{tabular}

Fuente: propia de los investigadores.

\begin{tabular}{|c|c|c|c|c|c|c|}
\hline \multicolumn{7}{|c|}{ SOMETIMIENTO } \\
\hline & $\mathrm{n}$ & $\mu$ & $S$ & \multicolumn{2}{|c|}{ RANGO } & C.V.(\%) \\
\hline DÍA & 12 & 8.25 & 4.048 & 4 & 19 & 49.06676 \\
\hline NOCHE & 4 & 3.00 & 0.8165 & 2 & 4 & 27.21655 \\
\hline \multicolumn{7}{|c|}{ COPULA } \\
\hline & $\mathrm{n}$ & $\mu$ & $S$ & \multicolumn{2}{|c|}{ RANGO } & C.V.(\%) \\
\hline DÍA & 12 & 14.75 & 6.7302 & 4 & 26 & 45.62841 \\
\hline NOCHE & 4 & 28.50 & 3.873 & 23 & 32 & 13.58942 \\
\hline
\end{tabular}




\section{CONCLUSIONES}

- 12 vicuñas machos tardaron un promedio de 8'25" para poder someter a la hembra en horas del día. Esta fase en la noche es sumamente corta teniendo como resultado a 4 vicuñas macho que tardaron un promedio de 3'00" para someter a la hembra.

- En cuanto a la segunda fase para la copula o coito en horas del día, 12 vicuñas macho tardaron un, promedio de 14'75" y 4 vicuñas macho en horas de la noche tardaron en un promedio de 28'50"

- El tiempo del empadre para las 16 vicuñas en estudio fue: 12 vicuñas durante el día empadraron en un promedio total de 23' y para las 4 vicuñas restantes durante la noche fue de 31 '50".

- Estos tiempos son relacionados directamente con la quietud del medio y la presencia de otras vicuñas pudiendo ser estas: crías, madres y machos en el lugar del sometimiento y cópula.

\section{REFERENCIAS BIBLIOGRÁFICAS}

1. Novoa C., Flores A., 1991. Producción de rumiantes menores "Alpacas", Editorial Rumera Lima. Pp. $284-291$.

2. Riveros Fernández J. 2002 Avances en el Estudio de la Reproducción en Hembras de ungulados silvestres Universidad de Chile. 